\title{
MÓDULO DE ELASTICIDADE EM VIGAS DE MADEIRA DE DIMENSÕES ESTRUTURAIS PELO MÉTODO DOS MÍNIMOS QUADRADOS ${ }^{1}$
}

André Luis Christoforo², Júlio Cesar Molina ${ }^{3}$, Túlio Hallak Panzera ${ }^{4}$, Diego Henrique de Almeida ${ }^{5}$, Sérgio Luiz Moni Ribeiro Filho ${ }^{6}$, Ricardo de Mello Scaliante ${ }^{7}$ e Francisco Antonio Rocco Lahr ${ }^{3}$

\begin{abstract}
RESUMO - Este trabalho propõe uma metodologia alternativa de cálculo fundamentada no Método dos Mínimos Quadrados para a obtenção do módulo de elasticidade na flexão de vigas de madeira com dimensões estruturais. As equações desenvolvidas requerem o conhecimento de um ou cinco pontos de deslocamentos medidos ao longo do comprimento das peças. Esse método permite maior precisão sobre a variável resposta e tem como base o ensaio de flexão estática a três pontos. A metodologia proposta foi empregada em concordância com as recomendações da norma Brasileira NBR 7190:1997, adaptada para peças de dimensões estruturais. Os ensaios mecânicos foram realizados em sete peças de madeira Corymbia citriodora com $540 \mathrm{~cm}$ de comprimento, $15 \mathrm{~cm}$ de altura e $5 \mathrm{~cm}$ de largura. Em testes preliminares determinou-se o valor da força responsável por provocar um deslocamento vertical no meio do vão de L/200, sendo $L$ a distância entre os apoios das vigas. A força obtida foi dividida em sete incrementos iguais $(39,60 \mathrm{~N})$, fornecendo sete valores de deslocamento distintos. Os resultados de ambas as metodologias mostraram-se convergentes à medida que os deslocamentos se aproximaram da razão L/200, validando o uso desta relação. Os resultados do intervalo de confiança entre médias indicaram equivalência estatística entre os módulos de elasticidade por ambas as formas de cálculo e para os sete incrementos de deslocamentos. Em razão da possível presença de defeitos e da heterogeneidade da madeira, os resultados obtidos não devem ser extrapolados para outras madeiras de mesma ou de espécies diferentes, justificando o uso da presente metodologia de cálculo em cada pesquisa desenvolvida.
\end{abstract}

Palavras-chave: Madeira serrada; Módulo de elasticidade na flexão; Método dos Mínimos Quadrados.

\section{BENDING MODULUS OF ELASTICITY IN STRUCTURAL TIMBER BEAMS BY THE LEAST SQUARE METHOD}

\begin{abstract}
The objective of the present study was to propose an alternative method of calculation based on the Least Squares Method to determine the bending modulus of elasticity in timber beams with structural dimensions. The developed equations require the knowledge of one or five points of displacements along the length of the beam. This method allows greater reliability on the response variable and, it is based on the static three points bending tests. The proposed methodology was applied according to the recommendations of the Brazilian standard ABNT NBR 7190:1997, adapted for spans with structural dimensions. The tests were performed on seven Corymbia citriodora wood specimens with $540 \mathrm{~cm}$ of height, $15 \mathrm{~cm}$ of width and $5 \mathrm{~cm}$ of thickness. In preliminary tests was determined the value of the load responsible for causing a vertical
\end{abstract}

\footnotetext{
${ }^{1}$ Recebido em 08.08.2012 aceito para publicação em 03.09.2013.

${ }^{2}$ Departamento de Engenharia Mecânica da Universidade Federal de São joão del-Rei, UFSJ, MG, Brasil. E-mail: <alchristoforo@yahoo.com.br>.

${ }^{3}$ Departamento de Engenharia Mecânica da Universidade Federal de São joão del-Rei, UFSJ, MG, Brasil. E-mail: $<$ tuliopanzera@hotmail.com>.

${ }^{4}$ Escola de Engenharia de São Carlos da Universidade de São Paulo , EESC, USP, Brasil. E-mail: <juliocm@sc.usp.br> e $<$ frocco@sc.usp.br>.

${ }^{5}$ Programa de Pós-Graduação em Engenharia Civil na Universidade de São Paulo, USP, Brasil. E-mail: <almeidadh@us.br>. ${ }^{6}$ Programa de Graduação em Engenharia Mecânica na Universidade Federal de São João Del-Rei, UFSJ, Brasil. E-mail: <sergiolmrf@gmail.com>.

${ }^{7}$ Departamento Nacional de Infraestrutura de Transportes, Superintendência Regional do DNIT no Estado de Mato Grosso do Sul, Brasil. E-mail: <scaliante@usp.br>.
} 
displacement in the centre of the span (L/200), where $L$ is the distance between the support beams. The obtained load was divided into seven equal parts, with seven load increments of intensity $39.60 \mathrm{~N}$ and recorded the displacements corresponding to each of these load levels. The results of the confidence interval indicated statistical equivalence between the modulus of elasticity for both forms of calculation and for the seven increments of displacements. Due to the possible presence of defects and the heterogeneity of the wood, the results should not be extrapolated to other woods of the same or different species, justifying the use of this calculation method developed in each research.

Keywords: Sawn timber, Bending modulus of elasticity; Least Squares Method.

\section{INTRODUÇÃO}

Dentre os principais materiais utilizados na confecção de estruturas e elementos estruturais destaca-se a madeira, por ser um material de fonte renovável e de excelente relação resistência/peso, chegando a ser quatro vezes superior a do aço (CALIL et al., 2003). Além disso, ao contrário da crença popular, a madeira apresenta boa resistência ao fogo quando comparada com o aço.

O projeto de estruturas de madeira, assim como o de outros materiais, requer o conhecimento de algumas variáveis, dentre elas, o módulo de elasticidade, geralmente obtido por intermédio de ensaios experimentais destrutivos preconizados por documentos normativos, de maneira que os valores de forças e deslocamentos utilizados na obtenção da rigidez são baseados frações pré-estipuladas do valor de força máxima (resistência) obtida da experimentação, garantindo ser o módulo de elasticidade obtido em região elástica e linear.

Visando maior confiabilidade, por ser a madeira um material ortotrópico e heteronêneo, a sua caracterização na flexão requer experimentações em peças com dimensões estruturais. Neste âmbito, apenas documentos normativos internacionais podem ser citados, uma vez que a norma Brasileira ABNT NBR 7190:1997 (Projeto de Estruturas de Madeira), que trata da caracterização da madeira, contempla apenas a condição de ensaios destrutivos em corpos de prova de pequenas dimensões e isentos de defeitos para a determinação das propriedades de resistência e rigidez da madeira.

A norma ASTM D 198:1997 (Standard Test Method of Static Tests of Lumber in Structural Sizes) preconiza o ensaio de flexão estática a quatro pontos na determinação do módulo de elasticidade na flexão $\left(E_{m}\right)$, expresso pela Equação 1, sendo $F$ a força aplicada no limite de proporcionalidade elástico, $L$ a distância entre apoios, $a$ é distância entre as forças aplicadas, $b$ a largura do corpo de prova, $h$ a espessura do corpo de prova e $\ddot{a}$ a flecha obtida no meio do vão.

$$
E_{m}=\frac{F \cdot a \cdot\left(3 \cdot L^{2}-4 \cdot a^{2}\right)}{4 \cdot b \cdot h^{3} \cdot \delta}
$$

No Brasil, as pesquisas que envolvem a caracterização de peças de madeira de dimensões estruturais por intermédio de ensaios mecânicos seguem as premissas de métodos e cálculos contidos em documentos normativos internacionais, podendo-se citar os trabalhos de Pigozzo et al. (2000), Fiorelli (2005), Fiorelli et al. (2009); Miotto (2009) entre outros.

A caracterização de peças de madeira de dimensões estruturais também tem sido realizada por intermédio de ensaios não destrutivos, que visam à determinação das propriedades físicas e mecânicas de um elemento estrutural sem a alteração das suas capacidades de uso (ROSS et al., 1998). A vantagem do emprego de ensaios não destrutivos consiste na não necessidade da extração de corpos de prova, viabilizando o estudo da integridade estrutural (MINÁ et al., 2004; OLIVEIRA, SALES, 2002), comumente realizados por meio de ensaios como vibração transversal e ultra-som, entre outros.

Do exposto, os modelos de cálculo propostos por documentos normativos para a determinação do módulo de elasticidade na flexão em peças de madeira de dimensões estruturais não contemplam metodologias de otimização, sendo obtidos por meio de ensaios destrutivos.

Este trabalho propõe uma metodologia analítica de cálculo, fundamentada no Método dos Mínimos Quadrados e no ensaio de flexão estática a três pontos, conduzido de forma não destrutiva, para a determinação do módulo de elasticidade à flexão em peças de madeira serrada de dimensões estruturais. O emprego da abordagem analítica proposta é feito para madeiras

Revista Árvore, Viçosa-MG, v.37, n.5, p.981-988, 2013

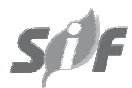


da espécie Corymbia citriodora, sendo os resultados dos módulos de elasticidade obtidos comparados com os oriundos da Norma Brasileira ABNT NBR 7190:1997, adaptada para a condição de peças de dimensões estruturais e ensaios não destrutivos, permitindo-se verificar as diferenças entre ambos.

\section{MATERIAIS E MÉTODOS}

Para a determinação do módulo de elasticidade foram utilizadas 7 peças de madeira da espécie Corymbia citriodora com $540 \mathrm{~cm}$ de comprimento, $15 \mathrm{~cm}$ de altura e $5 \mathrm{~cm}$ de largura, respeitando a relação $\mathrm{L} \leq 21 \mathrm{~h}$ (ROCCO LHAR, 1983), desprezando-se assim os efeitos do cisalhamento no cálculo das deflexões das peças. Os ensaios experimentais utilizados na determinação dos módulos de elasticidade são considerados não destrutivos, em virtude dos maiores valores em deslocamentos encontrados estarem limitados a relação L/200 (não baseados no valor da força de ruptura), sendo esta uma media de pequenos deslocamentos definida pela norma ABNT NBR 7190:1997, implicando em linearidade física e geométrica para as vigas de madeira testadas.

Os esquemas dos ensaios estruturais para a obtenção dos módulos de elasticidade das vigas por ambas as metodologia de cálculo são apresentados na Figura 1, sendo estes executados nas dependências do Laboratório de Estruturas de Madeira (LaMEM) do Departamento de Engenharia de Estruturas (SET) da Escola de Engenharia de São Carlos (EESC) da Universidade de São Paulo (USP).

O esquema de ensaio ilustrado na Figura 1a (um relógio comparador) foi utilizado para o cálculo do módulo de elasticidade pela norma Brasileira ABNT NBR 7190:1997 (adaptado para peças com dimensões estruturais) assim como para o emprego de uma dentre duas abordagens analíticas de cálculo fundamentadas no Método dos Mínimos Quadrados (abordagem alternativa). O esquema ilustrado pela Figura 1c é utilizado para o desenvolvimento da segunda abordagem alternativa de cálculo (cinco relógios comparadores), sendo medidos os deslocamentos em cinco pontos distintos ao longo das peças.

\subsection{Metodologia alternativa com o uso de um relógio comparador}

A equação para o cálculo do módulo de elasticidade na flexão com o uso de um único relógio comparador $\left(E_{e f}\right)$ é aqui apresentada para dois níveis de força e deslocamentos obtidos da experimentação, sendo generalizada posteriormente para três ou mais níveis.

Da mecânica dos sólidos, o deslocamento $(\delta)$ no ponto médio da viga do modelo estrutural ilustrado na Figura 1a é expresso pela Equação 2, sendo $E$ o módulo de elasticidade longitudinal ou de Young e $b$ e $h$ as medidas da largura e altura da seção transversal da peça respectivamente (Figura 1a).

$$
\delta=\frac{F \cdot L^{3}}{4 \cdot E \cdot b \cdot h^{3}}
$$

Definindo-se as duas medidas sucessivas de forças $F_{1}$ e $F_{2}$ relacionadas aos deslocamentos $\delta_{1}$ e $\delta_{2}$ obtidos experimentalmente, os deslocamentos analíticos para estas forças ficam determinados pelas Equações 3 e

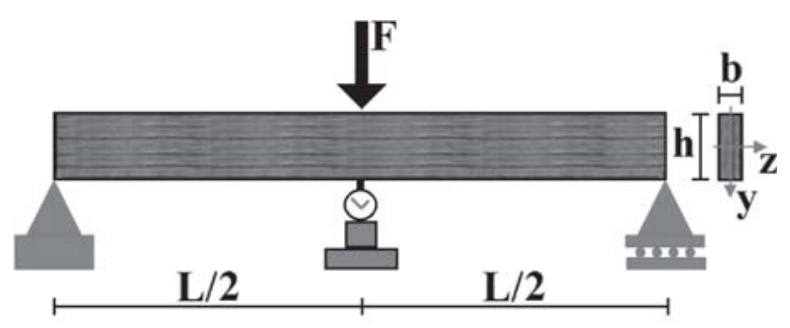

(a)



(b)

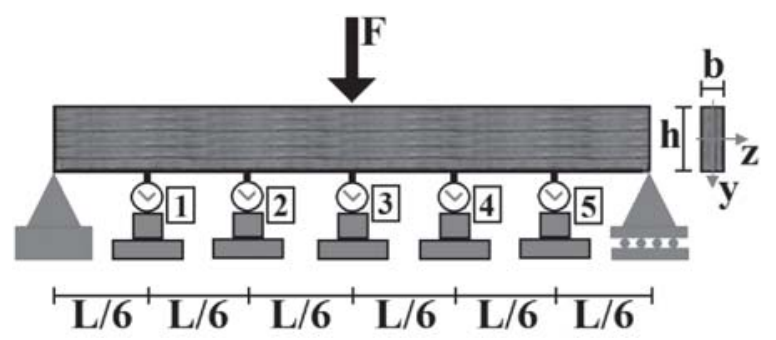

(c)

Figura 1 - Esquemas dos ensaios para obtenção dos módulos de elasticidade.

Figure 1 - Schemes tests to obtain the modulus of elasticity.

Revista Árvore, Viçosa-MG, v.37, n.5, p.981-988, 2013 
4 respectivamente, escritos como função do módulo de elasticidade.

$$
\begin{gathered}
\delta_{1}(E)=\frac{1}{E} \cdot \frac{F_{1} \cdot L^{3}}{4 \cdot b \cdot h^{3}} \\
\delta_{2}(E)=\frac{1}{E} \cdot \frac{F_{2} \cdot L^{3}}{4 \cdot b \cdot h^{3}}
\end{gathered}
$$

A presente metodologia fundamenta-se na ideia de mínimos quadrados (Equação 5), objetivando-se determinar o valor do módulo de elasticidade para que o resíduo gerado entre os valores dos deslocamentos analíticos $\left(\delta(E)_{i}\right)$ e experimentais $\left(\delta_{i}\right)$ seja o menor possível.

$$
f(E)=\frac{1}{2} \cdot \sum_{i=1}^{n}\left(\delta(E)_{i}-\delta_{i}\right)^{2}
$$

Substituindo-se as Equações 3 e 4 na Equação 5 e derivando-se esta última e igualando-a a zero chegase ao valor do módulo de elasticidade $\left(E_{m, e f}\right)$ que minimiza as diferenças entre os deslocamentos analíticos e experimentais (Equação 6), comprovando ser este ponto de mínimo global pelo critério da derivada segunda.

$$
E_{e f, 2}=\frac{\left(F_{1}^{2}+F_{2}^{2}\right) \cdot L^{3}}{4 \cdot b \cdot h^{3} \cdot\left(F_{1} \cdot \delta_{1}+F_{2} \cdot \delta_{2}\right)}
$$

O módulo de elasticidade para três e quatro níveis de forças e deslocamentos sucessivos medidos experimentalmente são expressos pelas Equações 7 e 8 .

$$
\begin{gathered}
E_{e f, 3}=\frac{\left(F_{1}^{2}+F_{2}^{2}+F_{3}^{2}\right) \cdot L^{3}}{4 \cdot b \cdot h^{3} \cdot\left(F_{1} \cdot \delta_{1}+F_{2} \cdot \delta_{2}+F_{3} \cdot \delta_{3}\right)} \\
E_{e f, 4}=\frac{\left(F_{1}^{2}+F_{2}^{2}+F_{3}^{2}+F_{4}^{2}\right) \cdot L^{3}}{4 \cdot b \cdot h^{3} \cdot\left(F_{1} \cdot \delta_{1}+F_{2} \cdot \delta_{2}+F_{3} \cdot \delta_{3}+F_{4} \cdot \delta_{4}\right)}
\end{gathered}
$$

Para um conjunto de $n$ forças e deslocamentos sucessivos obtidos experimentalmente, o módulo de elasticidade fica determinado pela Equação 9.

$$
E_{e f, n}=\frac{L^{3}}{4 \cdot b \cdot h^{3}} \cdot \frac{\sum_{i=1}^{n}\left(F_{i}^{2}\right)}{\sum_{i=1}^{n}\left(\delta_{i} \cdot F_{i}\right)}
$$

\subsection{Metodologia alternativa com o uso de cinco relógios comparadores}

A metodologia utilizada no cálculo do módulo de elasticidade ótimo $\left(E_{o t m}\right)$ é apresentada para a condição de três relógios comparadores, sendo análoga para a condição de cinco.

Da resistência dos materiais, os deslocamentos nos relógios comparadores 1 e 3 (Figura 1b) são expressos pela Equação 10, sendo o deslocamento no relógio comparador 2 obtido da Equação 2, ambos escritos como função do módulo de elasticidade.

$$
\delta_{13}=\delta=\frac{11 \cdot F \cdot L^{3}}{768 \cdot E \cdot I_{Z}}
$$

O módulo de elasticidade a ser calculado com as informações advindas do modelo de ensaio da Figura $1 \mathrm{~b}$, assim como apresentado na metodologia envolvendo 1 relógio comparador, parte da idéia de mínimos quadrados (Equação 5), sendo utilizado os valores dos deslocamentos analíticos das Equações 2 e 10. Derivando-se a equação resultante e igualando-a a zero chega-se ao valor do módulo de elasticidade ótimo (Equação 11).

$$
E_{\text {otm }}=\frac{249 \cdot F \cdot L^{3}}{32 \cdot \gamma \cdot b \cdot h^{3}}, \gamma=11 \cdot\left(\delta_{1}+\delta_{3}\right)+16 \cdot \delta_{2}
$$

Utilizando-se da mesma metodologia para o cálculo da Equação 11 chega-se a expressão para o cálculo do módulo de elasticidade na flexão com o uso de cinco relógios comparadores (Figura 1c), expressa pela Equação 12.

$$
E_{\text {otm }}=\frac{2125 \cdot F \cdot L^{3}}{108 \cdot \beta \cdot b \cdot h^{3}},\left\{\begin{aligned}
\beta=13 \cdot \gamma_{1} & +23 \cdot \gamma_{2}+27 \cdot \delta_{3} \\
\gamma_{1} & =\delta_{1}+\delta_{5} \\
\gamma_{2} & =\delta_{2}+\delta_{4}
\end{aligned}\right.
$$

A Equação 2 reescrita em função do módulo de elasticidade $\left(E_{s t}\right)$ é a equação adaptada da Norma Brasileira, consistindo em uma abordagem simplificada. Esta juntamente com as Equações 9 e 12são utilizadas no cálculo dos módulos de elasticidade das peças de madeira de dimensões estruturais.

Em um teste inicial, determinou-se um valor de força de $277 \mathrm{~N}$ responsável por provocar um deslocamento próximo a L/200. Este valor foi dividido em sete incrementos de carga, conferindo sete medições em forças e deslocamentos por viga ensaiada, conduzindo, para cada uma das três formas de análise, a sete valores 
de módulo de elasticidade por peça. Ressalta-se que cada peça foi ensaiada uma única vez na flexão, sendo os módulos de elasticidade obtidos dos sucessivos e progressivos incrementos de carga, visto o modelo estrutural com cinco relógios contemplar também aquele com o uso de apenas um relógio comparador.

Para verificar as diferenças entre os valores dos módulos de elasticidade obtidos da norma Brasileira com os advindos das duas abordagens alternativas de cálculo foi-se utilizado o intervalo de confiança da diferença entre médias, expresso pela Equação 13, sendo $m$ a média populacional das diferenças, $\bar{x}_{m}$ a média aritmética amostral das diferenças, $n$ o tamanho da amostra, $S_{m}$ o desvio padrão amostral das diferenças e $t_{\alpha / 2, n-1}$ o valor tabelado pela distribuição t de Student com $n$-1 graus de liberdade e nível de significância $\alpha(5 \%)$.

$$
\bar{x}_{m}-t_{\alpha / 2, n-1} \cdot S_{m} / \sqrt{n} \leq \mu \leq \bar{x}_{m}+t_{\alpha / 2, n-1} \cdot S_{m} / \sqrt{n}
$$

\section{RESULTADOS}

A Tabela 1 apresenta os valores dos módulos de elasticidade obtidos da abordagem adaptada da norma
Brasileira (simplificada) e das metodologias alternativas com um e cinco relógios comparadores respectivamente.

Observando-se a variação dos módulos de elasticidade para ambas as metodologias de cálculo, nota-se em cada caso uma sequência descendente nos valores, indicando convergência à medida que os deslocamentos no meio do vão se aproximam da medida L/200, assim como ilustra a Figura 2.

As Tabelas 2 e 3 exibem o teste de hipótese ou itervalo de confiança das médias de duas populações independentes, avaliando-se a equivalência estatística entre os módulos de elasticidade entre a abordagem simplificada $\left(\mathrm{E}_{\mathrm{st}}\right)$ e alternativa com o uso de um relógio comparador $\left(\mathrm{E}_{\mathrm{ef}}\right)$, e entre a metodologia simplificada e a alternativa com o uso de cinco relógios comparadores $\left(\mathrm{E}_{\mathrm{otm}}\right)$ respectivamente. Os intervalos de confiança associados a P-valores superiores a 0,05 (5\% de significância) são considerados estaticamente equivalentes. (WERKEMA; AGUIAR, 1996).

Através do intervalo de confiança, pode-se constatar, com 95\% de confiança, a equivalência estatística entre os módulos de elasticidade obtidos

Tabela 1 - Módulos de elasticidade (MPa).

Table 1 - Modulus of elasticity (MPa).

\begin{tabular}{|c|c|c|c|c|c|c|c|}
\hline & Viga 1 & Viga 2 & Viga 3 & Viga 4 & Viga 5 & Viga 6 & Viga 7 \\
\hline$E_{\text {st, }}$ & 2287 & 2849 & 2914 & 2789 & 2893 & 2839 & 2573 \\
\hline $\mathbf{E}_{\mathrm{st}, 2}$ & 2167 & 2749 & 2791 & 2533 & 2533 & 2352 & 2195 \\
\hline $\mathbf{E}_{\mathrm{st}, 3}^{\mathrm{st}, 2}$ & 2148 & 2724 & 2470 & 2058 & 2352 & 2375 & 2008 \\
\hline$E_{\text {st, } 4}$ & 2138 & 2659 & 2352 & 1882 & 2352 & 2352 & 1960 \\
\hline $\mathbf{E}_{\mathrm{st}, 5}^{\mathrm{st,4}}$ & 2111 & 2549 & 2287 & 1790 & 2287 & 2352 & 1906 \\
\hline$E_{s t, 6}$ & 2148 & 2475 & 2205 & 1703 & 2245 & 2352 & 1885 \\
\hline \multirow[t]{2}{*}{$\mathbf{E}_{\mathrm{st}, 7}$} & 2119 & 2381 & 2175 & 1646 & 2217 & 2352 & 1853 \\
\hline & Viga 1 & Viga 2 & Viga 3 & Viga 4 & Viga 5 & Viga 6 & Viga 7 \\
\hline$E_{\text {ef, } 1}$ & 2274 & 2956 & 2938 & 2929 & 2956 & 2680 & 2728 \\
\hline $\mathbf{E}_{\mathrm{ef}, 2}$ & 2221 & 2844 & 2838 & 2747 & 2632 & 2521 & 2259 \\
\hline $\mathbf{E}_{\mathrm{ef}, 3}$ & 2117 & 2506 & 2531 & 2108 & 2429 & 2236 & 2015 \\
\hline$E_{e f, 4}$ & 2076 & 2448 & 2406 & 1953 & 2339 & 2341 & 1941 \\
\hline$E_{e f, 5}$ & 2078 & 2305 & 2343 & 1822 & 2209 & 2331 & 1902 \\
\hline$E_{e f, 6}$ & 2114 & 2052 & 2290 & 1744 & 2185 & 2301 & 1873 \\
\hline \multirow[t]{2}{*}{$\mathbf{E}_{\text {ef }, 7}$} & 2113 & 1905 & 2254 & 1691 & 2180 & 2235 & 1841 \\
\hline & Viga 1 & Viga 2 & Viga 3 & Viga 4 & Viga 5 & Viga 6 & Viga 7 \\
\hline$E_{\text {otm, } 1}$ & 21463 & 29667 & 29452 & 27827 & 26032 & 23876 & 22170 \\
\hline $\mathbf{E}_{\text {otm, }, 2}$ & 21197 & 27939 & 25853 & 22372 & 24038 & 20488 & 20504 \\
\hline $\mathbf{E}_{\mathrm{otm}, 3}$ & 21070 & 24622 & 24283 & 20091 & 23505 & 20256 & 19795 \\
\hline$E_{\text {otm }, 4}$ & 20897 & 22085 & 23397 & 18847 & 22997 & 20165 & 19272 \\
\hline $\mathbf{E}_{\text {otm, }, 5}$ & 20891 & 21957 & 22659 & 17933 & 22596 & 20122 & 18951 \\
\hline$E_{o t m, 6}$ & 20870 & 20708 & 22170 & 17262 & 22284 & 20099 & 18668 \\
\hline $\mathbf{E}_{\mathrm{otm}, 7}$ & 20770 & 20175 & 21845 & 16783 & 22038 & 20034 & 18464 \\
\hline
\end{tabular}


com o uso da metodologia alternativa com os advindos da abordagem simplificada adaptada da norma Brasileira ABNT NBR 7190:1997.
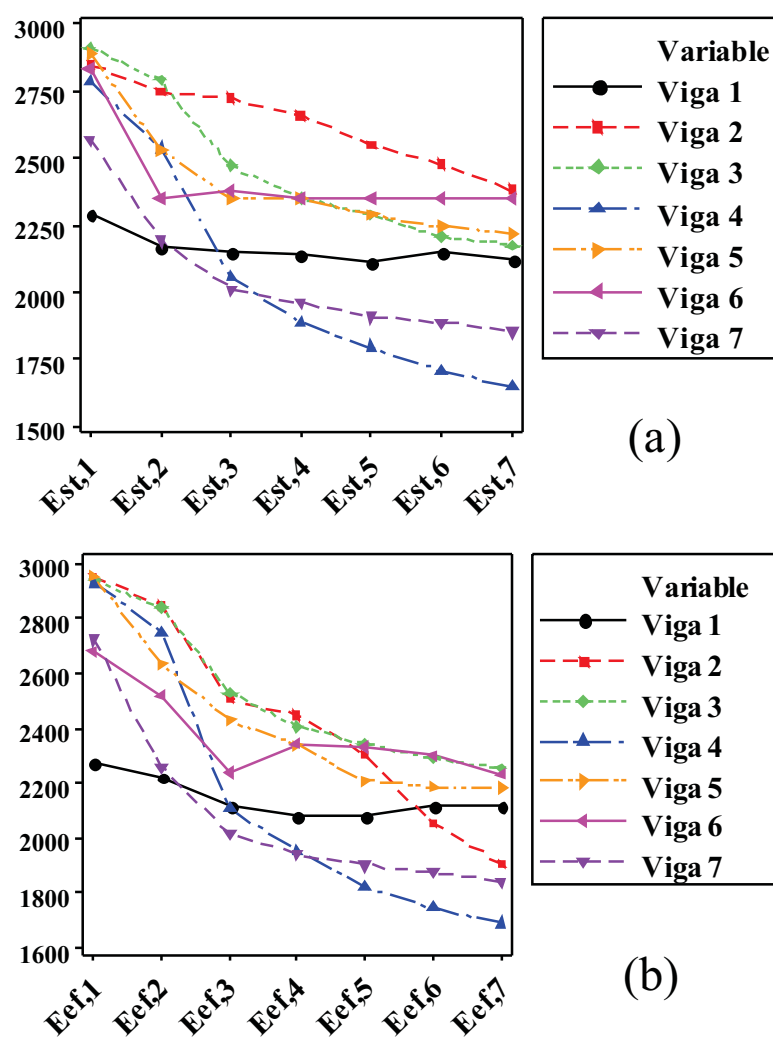

(b)

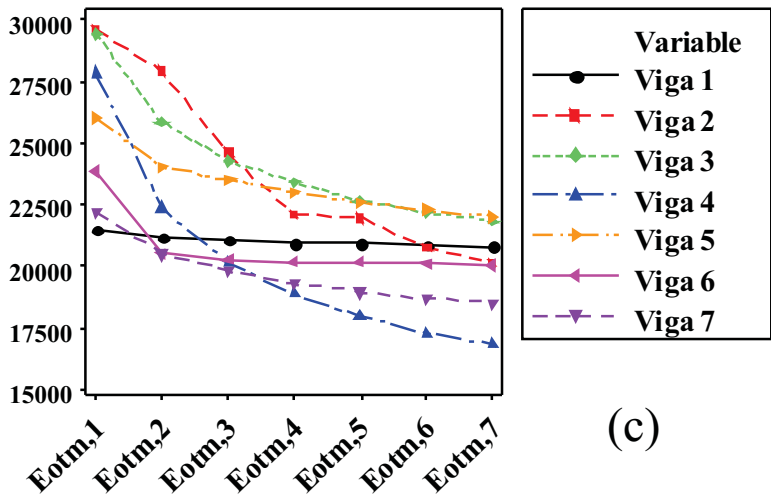

Figura 2 - Sequência de valores dos módulos de elasticidade: (a)Abordagem simplificada, (b) Abordagem alternativa com um relógio comparador e (c) Abordagem alternativa com cinco relógios comparadores.

Figure 2 - Sequence of values of the modulus of elasticity: (a) Simplified approach, (b) Alternative approach with one dial gauge and (c) Alternative approach with five dial gauges.

\section{DISCUSSÕES}

A equivalência estatística entre os módulos de elasticidade oriundos de ambas as metodologias de cálculo se justificam principalmente pela não existência de defeitos nas peças ensaiadas, possivelmente detectados com o uso da metodologia alternativa com o uso de cinco relógios comparadores.

Os resultados obtidos com o uso da Equação 9 $\left(\mathrm{E}_{\mathrm{st}}\right)$ evidenciaram que para medidas de deslocamentos iguais e inferiores a razão $L / 200$, ainda sim os módulos de elasticidade por ambas as formas de cálulo foram equivalentes, revelando ser ainda viável o uso de outros limites de deslocamentos para obtenção da rigidez das peças testadas.

\section{CONCLUSÕES}

A presente metodologia permite a obtenção do módulo de elasticidade na flexão em vigas de madeira de dimensões estruturais com maior precisão quando comparada as metodologias de cálculo presentes nos documentos normativos, por estar fundamentada em mínimos quadrados, possibilitando o emprego de várias medidas entre forças e deslocamentos sucessivos.

Os sete incrementos de força e desloamentos utilizados em cada peça e por cada metodologia de cálculo possibilitou avaliar a convergência nos valores dos módulos de elasticidade, comprovando a validade da medida em deslocamentos $L / 200$ (estado limite de utilização) estabelecida pela norma Brasielira, assim como da validade de medidas de deslocamentos inferiores a estabelecida por este código normativo.

Os resultados do intervalo de confiança revelaram equivalência estatística entre os módulos de elasticidade oriundos da abordagem fundamentada em mínimos quadrados para com a adaptada da norma Brasileira, indicando a boa aproximação do modelo adaptado da norma para o cálculo do módulo de elasticidade na flexão das vigas avaliadas. Ressalta-se que a possível existência de defeitos e a heterogeneidade nas peças de madeira testadas podem afetar de forma significativa o cálculo do módulo de elasticidade. Dessa forma, os resultados obtidos não podem ser extrapolados para madeiras de mesma ou de espécies diferentes, justificando o emprego das abordagens alternativas de cálculo em cada estudo desenvolvido, principalmete a que faz uso 
Tabela 2 - Teste de hipóteses entre os módulos de elasticidade $\mathrm{E}_{\mathrm{st}}$ e $\mathrm{E}_{\mathrm{ef}}$. Table 2 - Hypothesis tests between the modulus of elasticity $E_{s t}$ and $E_{e f}{ }^{\circ}$

\begin{tabular}{|c|c|c|c|c|c|}
\hline \multicolumn{2}{|c|}{ Teste de hipóteses } & \multirow{2}{*}{$\begin{array}{c}\text { Desvio Padrão } \\
59,2 \\
75,7\end{array}$} & \multirow{2}{*}{$\begin{array}{l}\text { Média } \\
2159,7 \\
2141,3\end{array}$} & \multirow{2}{*}{$\frac{\text { P-valor }}{0,633}$} & \multirow{2}{*}{$\begin{array}{c}\text { Intervalo de confiança } \\
-62,0785 \leq \mu \leq 97,7928\end{array}$} \\
\hline Teste $\mathrm{t}$ : & $\begin{array}{l}E_{s t, 1} \\
E_{e f, 1}\end{array}$ & & & & \\
\hline Teste $\mathrm{t}$ : & $\begin{array}{l}E_{\text {st }, 2} \\
E_{e f, 2}\end{array}$ & $\begin{array}{l}165 \\
385\end{array}$ & $\begin{array}{l}2627 \\
2431\end{array}$ & 0,251 & $-169,349 \leq \mu \leq 560,777$ \\
\hline Teste $\mathrm{t}$ : & $\begin{array}{l}E_{\text {st, } 3} \\
E_{e f, 3}\end{array}$ & $\begin{array}{l}290 \\
272\end{array}$ & $\begin{array}{l}2456 \\
2514\end{array}$ & 0,707 & $-388,539 \leq \mu \leq 272,53$ \\
\hline Teste $\mathrm{t}$ : & $\begin{array}{l}E_{s t, 4} \\
E_{e f, 4}\end{array}$ & $\begin{array}{l}439 \\
498\end{array}$ & $\begin{array}{l}2057 \\
2142\end{array}$ & 0,742 & $-637,0556 \leq \mu \leq 467,62$ \\
\hline Teste $\mathrm{t}$ : & $\begin{array}{l}E_{s t, 5} \\
E_{e f, 5}\end{array}$ & $\begin{array}{l}236 \\
288\end{array}$ & $\begin{array}{l}2411 \\
2419\end{array}$ & 0,960 & $-316,8709 \leq \mu \leq 302,29$ \\
\hline Teste $\mathrm{t}:$ & $\begin{array}{l}E_{\text {st, } 6} \\
E_{e f, 6}\end{array}$ & $\begin{array}{l}183 \\
164\end{array}$ & $\begin{array}{l}2425 \\
2378\end{array}$ & 0,623 & $-157,42 \leq \mu \leq 251,42$ \\
\hline Teste $\mathrm{t}$ : & $\begin{array}{l}E_{\text {st, } 7} \\
E_{e f, 7}\end{array}$ & $\begin{array}{l}255 \\
318\end{array}$ & $\begin{array}{l}2054 \\
2080\end{array}$ & 0,871 & $-364,885 \leq \mu \leq 313,74$ \\
\hline
\end{tabular}

Tabela 3 - Teste de hipóteses entre os módulos de elasticidade $\mathrm{E}_{\mathrm{st}}$ e $\mathrm{E}_{\mathrm{otm}}$. Table 3 - Hypothesis tests between the modulus of elasticity $E_{s t}$ e $E_{o t m}$.

\begin{tabular}{|c|c|c|c|c|c|}
\hline \multicolumn{2}{|c|}{ Teste de hipóteses } & \multirow{2}{*}{$\begin{array}{c}\text { Desvio Padrão } \\
4573 \\
240\end{array}$} & \multirow{2}{*}{$\begin{array}{l}\text { Média } \\
20887 \\
21023\end{array}$} & \multirow{2}{*}{$\begin{array}{c}\text { P-valor } \\
0,940\end{array}$} & \multirow{2}{*}{$\begin{array}{l}\text { Intervalo de confiança } \\
-4370,87 \leq \mu \leq 4099,715\end{array}$} \\
\hline Teste $\mathrm{t}$ : & $\begin{array}{c}E_{s t, 1} \\
E_{o t m, 1}\end{array}$ & & & & \\
\hline Teste t: & $\begin{array}{c}E_{s t, 2} \\
E_{o t m, 2}\end{array}$ & $\begin{array}{l}4573 \\
3679\end{array}$ & $\begin{array}{c}208877 \\
23879\end{array}$ & 0,205 & $-7874,79 \leq \mu \leq 1890,79$ \\
\hline Teste $\mathrm{t}$ : & $\begin{array}{c}E_{\mathrm{st}, 3} \\
\mathrm{otm}, 3\end{array}$ & $\begin{array}{l}4573 \\
2677\end{array}$ & $\begin{array}{l}20887 \\
24237\end{array}$ & 0,129 & $-7880,79 \leq \mu \leq 1180,79$ \\
\hline Teste $\mathrm{t}$ : & $\begin{array}{c}E_{s t, 4} \\
E_{o t m, 4}\end{array}$ & $\begin{array}{l}4573 \\
3877\end{array}$ & $\begin{array}{l}20887 \\
20159\end{array}$ & 0,754 & $-4259,583 \leq \mu \leq 5715,012$ \\
\hline Teste $\mathrm{t}$ : & $\begin{array}{c}E_{s t, 5} \\
E_{o t m, 5}\end{array}$ & $\begin{array}{l}4573 \\
1369\end{array}$ & $\begin{array}{l}20887 \\
23356\end{array}$ & 0,214 & $-6735,08 \leq \mu \leq 1797,65$ \\
\hline Teste $\mathrm{t}$ : & $\begin{array}{c}E_{s t, 6} \\
E_{o t m, 6}\end{array}$ & $\begin{array}{l}4573 \\
1399\end{array}$ & $\begin{array}{l}20887 \\
20720\end{array}$ & 0,929 & $-4107,314 \leq \mu \leq 4441,314$ \\
\hline Teste $\mathrm{t}$ : & $\begin{array}{c}E_{s t, 7} \\
E_{o t m, 7}\end{array}$ & $\begin{array}{l}4573 \\
1297\end{array}$ & $\begin{array}{l}20887 \\
19689\end{array}$ & 0,530 & $-3198,39 \leq \mu \leq 5594,10$ \\
\hline
\end{tabular}

dos cinco relógios comparadores, sendo foco de pesquisas futuras visando investigar a influência de defeitos em vigas na obtenção da rigidez.

\section{REFERÊNCIAS}

AMERICAN SOCIETY FOR TESTING AND MATERIALS - ASTM. Standard test method of static tests of lumber in structural sizes. ASTM D 198. Annual book of ASTM standards, v. 03.01. West Conshohocken, Philadelphia: 1997.
ASSOCIAÇÃO BRASILEIRA DE NORMAS TÉCNICAS - ABNT. Projeto de estruturas de madeira. NBR 7190. Rio de Janeiro: 1997.

CALIL JR., C.; LAHR, F. A. R.; DIAS, A. A. Dimensionamento de elementos estruturais de madeira. Barueri: Manole, 2003.

FIORELLI, J. Estudo teórico e experimental de vigas de madeira laminada colada reforçadas com fibra de vidro. 2005. 108f. Tese (Doutorado em Ciência e Engenharia de Materiais) - Universidade de São Paulo, São Carlos, 2005.

Revista Árvore, Viçosa-MG, v.37, n.5, p.981-988, 2013 
FIORELLI, J.; DIAS, A. A.; COIADO, B. Propriedades mecânicas de peças com dimensões estruturais de Pinus spp: correlação entre resistência à tração e classificação visual. Revista Árvore, v.33, n.4, p.741750, 2009.

MINÁ, A. J. S. et al. Avaliação não destrutiva de postes de madeira por meio de ultrassom. Scientia Forestalis, n.65, p.188-196, 2004.

MIOTTO, J. L.; DiAS, A. A. Produção e avaliação de vigas de madeira laminada colada confeccionadas com lâminas de eucalipto. Revista Tecnológica, Edição Especial ENTECA, p.35-45, 2009.

OLIVEIRA, F. G. R.; SALES, A. Ultrasonic measurements in Brazilian hardwood.

Material Research, v.5, n.1, p.51-55, 2002.
PIGOZZO, J. C.; PLETZ, E.; LAHR, F. A. R. Aspectos da classificação mecânica de peças estruturais de madeira. In: ENCONTRO BRASILEIRO EM MADEIRAS E EM ESTRUTURAS DE MADEIRA, 7., 2000, São Carlos. Anais... São Carlos: 2000.

ROCCO LAHR, F. A. Sobre a determinação de propriedades de elasticidade da madeira. 1983. 216f. Tese (Doutorado em Engenharia de Estruturas) - Escola de Engenharia de São Carlos, Universidade de São Paulo, São Carlos, 1983.

ROSS, R. J.; BRASHAW, B. K.; PELLERIN, R. F. Nondestructive evaluation of wood. Forest Products Journal, v.48, n.1, p.14-19, 1998.

WERKEMA, M. C. C.; AGUIAR, S. Planejamento e análise de experimentos: como identificar e avaliar as principais variáveis influentes em um processo. Belo Horizonte: Fundação Christiano Ottoni, Escola de Engenharia da UFMG, 1996. 